Präv Gesundheitsf 2013 · 8:59-60

DOI 10.1007/s11553-013-0385-2

Online publiziert: 16. April 2013

(c) Springer-Verlag Berlin Heidelberg 2013

\title{
Marco Franze
}

Abteilung Versorgungsepidemiologie und Community Health, Institut für Community Medicine, Universitätsmedizin Greifswald, Greifswald, Deutschland

\section{Editorial}

den von der Agenda unmittelbar Betroffenen - diplomatisch ausgedrückt - eher nicht. Denn als eine der „Nebenwirkungen“" nennen die Agenda-Kritiker die Verschärfung sozialer Ungleichheit. Brückenschlag zur aktuellen Ausgabe der „Prävention und Gesundheitsförderung“:

Der soziale Gradient ist ebenfalls Thema des Beitrags von Stolzenberg et al., die in ihrem Beitrag „Wege zu Familien in sozial benachteiligten Regionen“ Ergebnisse der Evaluation des Projektes „Stadtteilmütter (STM) in Berlin-Kreuzberg" vorstellen. Das Projekt kann als erfolgreiches Beispiel zur Vermeidung des „Präventionsdilemmas“ betrachtet werden: So konnten im Zeitraum von etwa 2,5 Jahren durch die STM 953 Hausbesuche bei 118 Familien sowie 216 Einzelkontakte mit 191 Familien durchgeführt werden - und dies trotz der im Vorfeld nicht abzusehenden Zugangsschwierigkeiten. Das Setting Kommune ist ebenfalls Gegenstand des von mir zusammen mit Frau Gottschling-Lang und Herrn Prof. Hoffmann verfassten Beitrags zu Ergebnissen einer begleitenden Evaluation zum „Landesaktionsplan zur Gesundheitsförderung und Prävention MecklenburgVorpommern“. Dieser enthält gemeinsame Ziele und Handlungsschwerpunkte für gesundheitsfördernde und präventionsbezogene Aktivitäten in den Settings Kindertagesförderung, Schule, Betrieb/ Arbeitswelt und Kommune. Den Ergebnissen zufolge ist ein signifikanter Anstieg des Vernetzungsgrades zwischen den Akteuren und den Beratungsstellen im Land und den niedrigschwelligen aufsuchenden Angeboten zu berichten. Jedoch spielt dabei die Frage nach der Evidenz eine entscheidende Rolle, da die erhaltenen subjektiven Einschätzungen zukünftig in Bezug zu objektiven Daten gesetzt werden sollten.
Die Notwendigkeit von Wirksamkeitsstudien ist ein Ergebnis des Beitrags „Qualitätssicherung in der Gesundheitsförderung in Settings. Ansätze, Charakteristika und Empfehlungen“ von Tempel et al. Das dort beschriebene Projekt „Qualitätssicherung von Projekten zur Gesundheitsförderung in Settings “ zur Erstellung einer kriterienbasierten Übersicht über Instrumente und Verfahren der Qualitätssicherung in der Gesundheitsförderung lässt erkennen, dass nur wenige Instrumente und Verfahren evidenzbasiert sind und nur selten Informationen über Nutzung und Nutzen zur Verfügung gestellt werden.

Schmitt beschreibt in ihrem Beitrag „Gesundheitsförderung für Pflegeheimbewohner?" Ergebnisse leitfadengestützter qualitativer Interviews mit Pflegeheimbewohnern. Gerade psychosoziale und ethisch-moralische Maßnahmen zur Förderung von Gesundheit und Wohlbefinden im Pflegeheim erscheinen angezeigt, erfordern jedoch seitens der Pflegekräfte entsprechende Qualifikationen, insbesondere im Bereich sozial-kommunikativer und personaler Kompetenzen. Befasst sich Schmitt mit Menschen mit bereits bestehendem Pflegebedarf stellen Schaeffer und Horn in ihrem Beitrag die Prävention von Pflegebedürftigkeit in den Vordergrund. Die Ergebnisse ihrer explorativen Studie zur Perspektive von 15 Hausärzten in Ostwestfalen-Lippe verdeutlichen aufgrund von gehäuftem Auftreten chronischer Krankheiten und Multimorbidität die Notwendigkeit der Prävention von Pflegebedürftigkeit in Hausarztpraxen. Auch dieser Beitrag hebt die Bedeutsamkeit qualifikatorischer Aspekte hervor, um Präventionspotentiale hinreichend auszuschöpfen.

Wie der Beitrag von Stolzenberg et al. spielt die Frage nach dem Zugang zu spe- ern? Zumindest bei (ehemaligen) Anhängern der deutschen Sozialdemokratie und 
zifischen Zielgruppen im Beitrag „Was Dich nicht umbringt, macht Dich nur härter"von Martinz und Mir eine zentrale Rolle. Im Fokus: österreichische Grundwehrdiener, ihr Gesundheitszustand, -verhalten und ihr Interesse an gesundheitsförderlichen Maßnahmen. Den Autoren zufolge erweist sich das österreichische Bundesheer als aussichtsreiches Setting zur Implementierung solcher Interventionen.

Die individualisierte Sportberatung im Betrieb (namentlich die schweizerische Scintilla AG) und deren Effekte auf die Steigerung körperlicher Aktivität ist Gegenstand des Beitrags von Schmid et al.. Die Autoren zeigen Möglichkeiten auf, die evaluierte Intervention in die Betriebliche Gesundheitsförderung - auch unter Berücksichtigung verhältnispräventiver Maßnahmen - zu integrieren.

„Stress im Studium“ (so der Titel des Beitrags von Büttner u. Dlugosch) sowie die Rolle von Selbstwirksamkeitserwartungen und der Achtsamkeit im Stresserleben von Studierenden bilden den Fokus der Ergebnisse einer deutschlandweiten Onlinebefragung an über 2400 Teilnehmerinnen und Teilnehmern. Demnach erleben Studierende in hohem Maße Stress - dessen Reduktion und Prävention erscheint insbesondere unter Berücksichtigung der beiden genannten Konstrukte erforderlich.

Präventive Bonusprogramme und "Medical Savings Accounts“ als zwei ökonomische Anreizsysteme sind Gegenstand des Beitrags von Fischer („Ökonomische Anreize als Instrumente der Präventionspolitik“) und werden insbesondere vor dem Hintergrund ihrer positiven Anreize zur Steigerung von Präventionsbemühungen der Versicherten gegenüber gestellt. Fischer weist dabei insbesondere auf die Bedeutsamkeit der Förderung der intrinsischen Motivation und der Übernahme von Eigenverantwortung hin.

Die aktuelle Ausgabe der „Prävention und Gesundheitsförderung “ bietet Ihnen wieder eine Fülle spannender Beiträge. Daher wünsche ich Ihnen insbesondere viel Erkenntnisgewinn und übersende Ihnen aus Greifswald herzliche Grüße,

Ihr

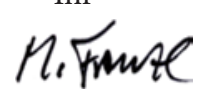

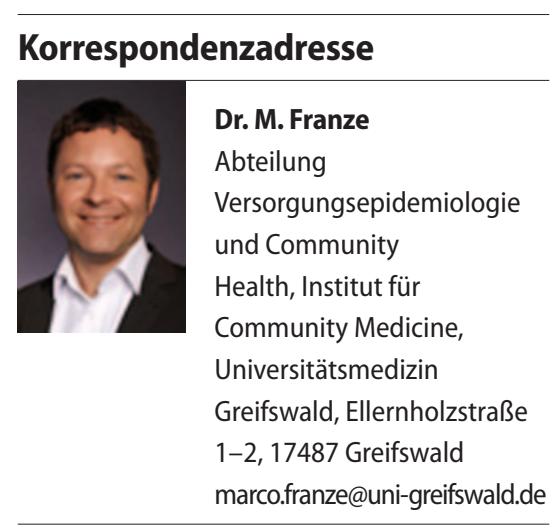

